Methods Patients with wide neck bifurcation aneurysm were included in WEBCAST2 if the aneurysm was not previously treated. An independent medical monitor independently analyzed adverse events. Follow-up imaging was obtained at 6 and 12 months up to 5 years after the procedure. An independent expert in Interventional Neuroradiology evaluated anatomical results using the 3 grades scale: complete occlusion, neck remnant, and aneurysm remnant.

Results From August 14 to May 15, 55 patients (38 females, 69.0\%) with 55 aneurysms including 4 ruptured (7.2\%) were included. Aneurysm locations were: middle cerebral artery in 25 patients (46.0\%), anterior communicating artery in 16 patients $(29.0 \%)$, basilar artery in 9 patients $(16.0 \%)$, and internal carotid artery terminus in 5 patients (9.0\%). Placement of the WEB device was feasible in 53/55 aneurysms (96.4\%). Forty-seven aneurysms were treated with the WEBSL and 6 with WEB-SLS. Adjunctive devices were used in 4 patients $(7.3 \%)$. Procedure-related morbidity and mortality at one month were reported in $1 / 54$ patients $(1.8 \%)$ and $0 / 54$ patients $(0.0 \%)$, respectively (1 patient was missing one-month follow-up).

Anatomical results at 6 months were complete aneurysm occlusion in 28/49 aneurysms (57.1\%), neck remnant in 11/49 aneurysms (22.4\%), and aneurysm remnant in 10/49 aneurysms (20.4\%).

Conclusion Preliminary results of WEBCAST2 show similar safety and efficacy of WEB-SL/SLS compared to WEB-DL (WEBCAST) with low morbidity and no mortality and a high rate of adequate occlusion (79.5\%).

Disclosures L. Pierot: 2; C; Sequent. A. Molyneux: 2; C; Sequent. J. Byrne: 2; C; Sequent.

\section{0-021 FLOW DIVERSION IN RUPTURED INTRACRANIAL ANEURYSMS: A SYSTEMATIC REVIEW}

T Madaelil, C Moran, D Cross, A Kansagra. Neuroradiology, Mallinckrodt Institute of Radiology, Saint Louis, MO

\subsection{6/neurintsurg-2016-012589.21}

Background and purpose Flow diversion is an established technique to treat unruptured intracranial aneurysms not amenable to standard endovascular or microsurgical techniques. The suitability of flow diverting devices (FDD) to treat ruptured aneurysms is less clear.

Materials and methods An in-depth search of multiple electronic publication databases was performed for reports describing ruptured intracranial aneurysms treated by flow diversion. Clinical and radiological characteristics, interventional details, and outcomes were pooled and analyzed in aggregate.

Results The pooled cohort comprised of 126 patients with a mean age of $52.6 \pm 12.5$ years. Mean Hunt Hess/World Federation of Neurosurgical Societies grading scale at presentation was $2.2 \pm 1.2$. Treated aneurysms were located in the anterior circulation in 64\% (81/126) cases and 36\% (45/126) in the posterior circulation. Five distinct aneurysm morphologies were present, including dissecting (28\%, 35/125), fusiform (9.6\%, 12/125), giant $(3.2 \%, 4 / 125)$, blister $(37.6 \%, 47 / 125)$, and saccular $(21.6 \%, 27 / 125)$ types. Favorable clinical outcome (defined as Modified Rankin Scale (mRS) $=0-2$ or Glasgow Outcome Scale (GOS) $=4-5$ ) was achieved in $81.5 \%(101 /$ $124)$ of treated patients. Unfavorable clinic outcome (defined by $\mathrm{mRS}=3-6$ or GOS $=1-3)$ occurred in $18.5 \%(23 / 124)$ of patients. Clinically significant hemorrhagic complications occurred in $5.5 \%(7 / 126)$ of cases, the majority of which were due to aneurysm re-rupture $(4.8 \% ; 6 / 126)$. Aneurysm size greater than $2 \mathrm{~cm}$ was associated with a greater risk of re-rupture when compared to aneurysms smaller than $2 \mathrm{~cm}(\mathrm{p}=0.001)$. Aneurysm size greater than $7 \mathrm{~mm}$ was associated with more unfavorable clinical outcomes $(p=0.03)$.

Conclusion For ruptured aneurysms not amenable to other treatment strategies, the use of flow diversion may allow for reasonably high rates of good clinical outcomes, particularly in small aneurysms. There was a risk of re-rupture in these aneurysms, especially those larger than $2 \mathrm{~cm}$.

Disclosures T. Madaelil: None. C. Moran: 2; C; Medtronic Neurovascular. D. Cross: None. A. Kansagra: None.

\section{0-022 GENERAL ANESTHESIA, BASELINE ASPECTS, TIME TO TREATMENT, AND IV TPA IMPACT INTRACRANIAL HEMORRHAGE AFTER STENTRIEVER THROMBECTOMY: POOLED ANALYSIS FROM SWIFT PRIME, SWIFT AND STAR TRIALS}

${ }^{1} \mathrm{R}$ Raychev, ${ }^{2} \mathrm{~J}$ Saver, ${ }^{3} \mathrm{R}$ Jahan, ${ }^{4} \mathrm{R}$ Nogueira, ${ }^{5} \mathrm{M}$ Goyal, ${ }^{6} \mathrm{~V}$ Pereira, ${ }^{7} \mathrm{~J}$ Gralla, ${ }^{8} \mathrm{E}$ Levy, ${ }^{9} \mathrm{D}$ Yavagal, ${ }^{10} \mathrm{C}$ Cognard, ${ }^{11} \mathrm{D}$ Liebeskind. ${ }^{1}$ Saddleback Memorial Medical Center, Laguna Hills, CA; ${ }^{2}$ Neurology, UCLA Stroke Center, Los Angeles, CA; ${ }^{3}$ Division of Interventional Neuroradiology, UCLA, Los Angeles, CA; ${ }^{4}$ Marcus Stroke and Neuroscience Center, Grady Memorial Hospital, Department of Neurology, Emory University School of Medicine, Atalanta, GA; ${ }^{5}$ Departments of Radiology and Clinical Neurosciences, University of Calgary, Calgary, AB, Canada; ${ }^{6}$ Toronto Western Hospital/University of Toronto, Toronto, ON, Canada; ${ }^{7}$ Inselspital, University of Bern, Bern, Switzerland; ${ }^{8}$ Department of Neurosurgery, SUNY at Buffalo, Amherst, NY; ${ }^{9}$ University of Miami/Jackson Memorial Hospital, Miami, FL; ${ }^{10}$ Department of Diagnostic and Therapeutic Neuroradiology, University Hospital of Toulouse, Toulouse, France; "11 Neurovascular Imaging Research Core, Department of Neurology, UCLA, Los Angeles, California, USA, LOS Angeles, CA

\subsection{6/neurintsurg-2016-012589.22}

Introduction Despite the proven benefit of Solitaire for treatment of acute ischemic stroke, symptomatic intracranial hemorrhage (ICH) remains the most feared procedural complication. The aim of this analysis was to identify the factors determining $\mathrm{ICH}$ after neurothrombectomy with Solitaire stentriever.

Methods All patients $(\mathrm{N}=389)$ treated with Solitaire in SWIFT, SWIFT PRIME, and STAR trials were analyzed for incidence of 5 different ICH subtypes. Each ICH subtype was correlated with baseline clinical, imaging and procedural characteristic (age, NIHSS, hypertension, diabetes, atrial fibrillation, hyperglycemia, INR, platelet count, ASPECTS, general anesthesia, collateral grade, number of devices passes, final TICI, rescue therapy, IV TPA). Multivariate stepwise logistic regression model was used to identify the predictors of individual $\mathrm{ICH}$ subtypes.

Results ICH was observed in $21.6 \%(\mathrm{~N}=84)$ of which sICH was $1.0 \%(\mathrm{~N}=4)$, hemorrhage in ischemic territory (HIT) $19.3 \%(\mathrm{~N}=75), \mathrm{PH} 5.4 \%(\mathrm{~N}=21)$, and $\mathrm{SAH} 2.3 \%$ $(\mathrm{N}=9)$. The most significant predictors of any ICH, HIT, and $\mathrm{PH}$ are included in Table 1. No specific predictors of $\mathrm{SAH}$, and sICH were identified. Patients who achieved functional independence at 90 days (mRS 0-2) had significantly lower incidence of any ICH, HIT, PH, and no SICH (Table 2).

Conclusions Higher baseline ASPECTS, better collaterals and general anesthesia are associated with lower incidence of $\mathrm{ICH}$ after neurothrombectomy with Solitaire stentriever. Prolonged 